\title{
Mathematical modeling of electric consumption of the gas cooling process
}

\author{
Oybek Ishnazarov ${ }^{*}$, and Ural Hoshimov \\ Scientific and Technical Centre of JSC Uzbekenergo, Tashkent, Uzbekistan
}

\begin{abstract}
In the article, the possibility of determining the consumption of electrical energy by gas air cooling devices. Special attention is paid to the processing of experimental results. There are some assumptions in the mathematical model that do not affect the final result. The factors influencing the consumption of electric energy by gas air coolers are given. It is stated that the ambient temperature and gas pressure significantly affect the consumption of electrical energy. Thus, based on experimental data, a model of electrical energy consumption by gas air cooling devices of main compressor stations has been obtained.
\end{abstract}

\section{Introduction}

Transporting natural gas through main gas pipelines is a very complex and life-threatening process for operating personnel. In the process of gas transportation, the gas pressure increases for its further advance along the pipeline, which leads to an increase in the gas temperature at the outlet of the gas pipeline. The high gas temperature leads to the destruction of the gas pipeline's insulation coating and an increase in the supply of process gas, which increases energy costs. Gas cooling takes place in refrigeration units. Gas cooling units consist of a certain number of air coolers, which actuate elements in the system for maintaining a given gas temperature at the outlet of the compressor station. The task of maintaining the recommended gas temperature is solved by turning on the required number of fans.

In addition, the gas cooling unit is the main consumer of electricity at the gas turbine compressor station. Optimization of the operation of the electric drive of air coolers at the compressor station, optimization of the gas cooling system on the leg of the main gas pipeline will make it possible to select an optimizing mode of operation with minimal power consumption while maintaining the required throughput of the main gas pipeline and reducing the cost of transporting hydrocarbons.

In modern economic conditions at compressor stations, there is an acute issue of reducing the energy consumption of gas pumping while ensuring the specified quality indicators and volume. This problem cannot be solved without deep research based on mathematical modeling.

Therefore, this work aims to build a mathematical model of the consumption of electrical energy when cooling gas by air cooling devices. An apparatus for air cooling of gas is a

*Corresponding author: oybek.ishnazarov@gmail.com 
complex object in which various processes occur: aerodynamic and electromechanical, the process of heat exchange.

\section{Methods}

The authors in the article have analyzed the structure of electrical energy consumption by gas air coolers of main compressor stations. Experimental studies were carried out to determine electrical energy consumption by gas air coolers at main gas-pumping compressor stations. The most significant factors influencing the power consumption regime were identified.

Based on the results of the data obtained, an analysis of the factors that positively and negatively affect electrical energy consumption was carried out. To solve this goal, an analysis of the literature was carried out; experimental data were studied and summarized. Based on the theory of planning the experiment, the input and output parameters of electrical energy consumption were identified and selected. Further, based on the collection and analysis of experimental data, a mathematical model of the consumption of electrical energy of the gas cooling process was constructed.

As known, to reduce the consumption of electrical energy at compressor stations, including air-cooled units, a prerequisite is to take into account the changing parameters and appropriate regulation with a decrease in reservoir pressure and gas production volumes in the fields. This necessitates the use of two or more compression stages, replaceable flow paths of gas compressors, and a new unit on air coolers. In addition, an important condition is to determine the consumption of electrical energy depending on technological or other factors. At the same time, the possibility of reducing the consumption of electrical energy can reach up to $35 \%$. So in [1], the authors considered the processes occurring in the power supply system of the electric drive of the gas air cooling apparatus. In this work, based on the Rayleigh distribution, the analysis and assessment of the electrical energy consumption of air-cooled gas devices are presented. The fitted theoretical distribution was tested using the Kolmogorov goodness test and Pearson's statistics. Further, a mathematical model of the problem of a neural network method for predicting the power consumption of gas air cooling devices was developed for predicting the power consumption of gas air cooling devices was developed.

The authors of [2] investigated the possibilities of reducing energy consumption and increasing the efficiency of gas air cooling devices. The technology of energy-saving is presented, which is based on the use of a frequency-controlled electric drive of gas air cooling devices. Further, the method of thermal calculation of the gas air cooling apparatus was obtained, the change in the heat-power and aerodynamic characteristics of its operation was analyzed depending on the rotational speed of the electric motor rotor. An assessment of the economic efficiency of using a frequency-controlled electric drive has been carried out. Based on the assessment results, the authors proved the economic efficiency of frequency regulation of air coolers in the system under consideration.

Kolokov et al. [3] revealed the effect of the angle of installation of the fan blades of aircooled gas coolers on the temperature of the main gas outlet from the compressor station. The authors have developed a mathematical dependence of the gas temperature after air cooling devices on the gas temperature at the inlet to the devices and the ambient air temperature. It is shown that a change in the angle of inclination of the fan blades of gas air coolers will allow reducing not only the consumed electricity but also, with high-quality regulation of the outlet gas temperature, to reduce the fuel gas consumption at the subsequent compressor station. Further, the authors of the article presented a mathematical model of the power consumption of the devices from the angle of the blades. 
In [4], the technological features of gas temperature control in a two-stage design of the cooling section in air-cooled gas installations are investigated, an equation of the thermal efficiency is derived, a method for controlling a frequency-controlled drive is proposed, a method and an algorithm for calculating the optimal in terms of the energy-saving rotation frequency of electric drives of apparatus air cooling of the gas.

The article [5] defines the main performance indicators of gas cooling units at compressor stations of existing main gas pipelines. The technical and economic parameters of various structures of units in block-modular design are analyzed. A model and examples of calculating technical and economic indicators of methods for automatic control of air cooling devices are presented.

Molchalin et al. [6] assessed the relationship between the variables - electricity consumption, the throughput of the main gas pipeline, the gas temperature at the outlet of the compressor station. To solve the set tasks, the method of neural networks was used, which has several advantages over regression models: such networks themselves select the type of functional dependence according to experimental data and are an adaptive model that adjusts the network structure for new observations and makes it possible to explain rather complex relationships between power consumption and indicators of the main gas pipeline. In [7], based on analytical and experimental studies, a model was developed for calculating the optimal stationary operating modes of gas cooling units. The results achieved using various algorithms for controlling the rotational speed of the fan motors of air coolers are analyzed.

\section{Results and Discussion}

To select operating modes, build a model of electricity consumption, it is necessary to analyze the input and output streams of the gas cooling process. The results of experimental studies and the analysis of literature sources and industrial operation experience made it possible to determine that in the process of gas cooling, the main output parameter (controlled variable) is the gas temperature $\left(T,{ }^{0} \mathrm{C}\right)$. This parameter is controllable. The input parameters that determine the operating mode of gas air coolers are electrical energy $(\mathrm{W}, \mathrm{kW} \cdot \mathrm{h})$ and gas pressure $(p, P a)$. Outside temperature $\left(t,{ }^{0} \mathrm{C}\right)$ and relative humidity $(r$, $\%)$ of air serve as disturbing parameters. In addition, the amount of consumed electrical energy is influenced by both the quality parameters of the electrical energy itself and the gas pressure, relative humidity, air temperature, and gas temperature. To build a model of power consumption, authors [8] show the feasibility of considering the main technological factors.

Following this, Figure 1 shows the structure of the processes in the gas air cooling apparatus.

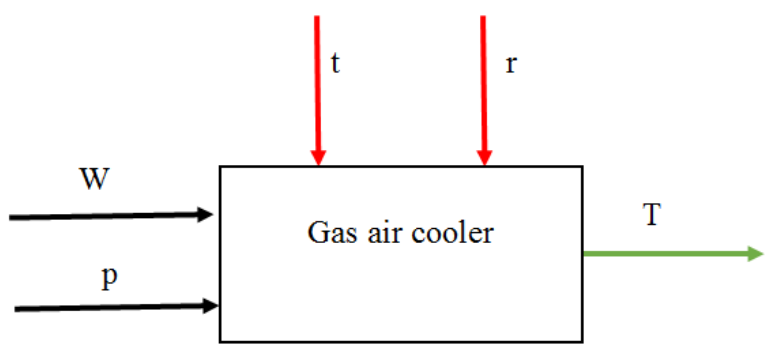

Fig. 1. The structure of processes in a gas air cooling apparatus 
Table 1 shows a generalized structure of the obtained experimental data. The measurements were carried out in June 2020 at a gas compressor station.

Table 1. Summary of Experimental Data

\begin{tabular}{|c|c|c|c|c|c|}
\hline № & $\begin{array}{c}\text { Outside } \\
\text { temperature, } \\
\text { t, }{ }^{0} \mathrm{C}\end{array}$ & $\begin{array}{c}\text { Relative } \\
\text { humidity, } \\
\text { r, \% }\end{array}$ & $\begin{array}{c}\text { Gas pressure, } \\
\text { p, } \mathrm{Pa}\end{array}$ & $\begin{array}{c}\text { Gas } \\
\text { temperature, } \\
\mathrm{T},{ }^{0} \mathrm{C}\end{array}$ & $\begin{array}{c}\text { Consumed } \\
\text { electrical } \\
\text { energy, } \\
\mathrm{W}, \mathrm{kWh}\end{array}$ \\
\hline 1 & 22.00 & 19.20 & 35.71 & 58.30 & 351 \\
\hline 2 & 22.00 & 19.30 & 35.62 & 58.40 & 352 \\
\hline 3 & 22.00 & 22.00 & 35.40 & 58.40 & 354 \\
\hline 4 & 27.00 & 21.50 & 35.51 & 58.41 & 409.5 \\
\hline 5 & 26.30 & 21.30 & 35.60 & 58.30 & 456 \\
\hline 6 & 28.70 & 19.90 & 35.64 & 58.90 & 514.2 \\
\hline 7 & 29.50 & 17.20 & 35.68 & 59.10 & 564.9 \\
\hline 8 & 30.60 & 14.90 & 35.71 & 60.20 & 632.3 \\
\hline 9 & 31.00 & 14.10 & 35.74 & 61.30 & 691 \\
\hline 10 & 32.90 & 13.40 & 35.81 & 61.80 & 745.9 \\
\hline 11 & 33.20 & 12.80 & 35.85 & 62.10 & 859.4 \\
\hline 12 & 33.90 & 12.00 & 35.92 & 62.40 & 972.8 \\
\hline 13 & 34.50 & 11.60 & 36.02 & 62.9 & 1068.6 \\
\hline 14 & 40 & 11.3 & 36.07 & 63.1 & 1105 \\
\hline 15 & 41.00 & 11.00 & 36.10 & 64.5 & 1183 \\
\hline 16 & 43 & 11.2 & 36.08 & 64.4 & 1382 \\
\hline 17 & 42.00 & 12.60 & 36.07 & 64.1 & 1241 \\
\hline 18 & 36.5 & 12.9 & 36.06 & 62.7 & 1064 \\
\hline 19 & 34.20 & 14.10 & 36.05 & 60.9 & 1027 \\
\hline 20 & 28 & 14.2 & 36.03 & 60.2 & 749.3 \\
\hline 21 & 27.00 & 14.60 & 36.00 & 59.2 & 694.2 \\
\hline 22 & 26 & 14.9 & 35.9 & 58.9 & 571.6 \\
\hline 23 & 25.50 & 16.20 & 35.80 & 58.6 & 522.3 \\
\hline 24 & 25 & 18.2 & 35.72 & 58.2 & 460.2 \\
\hline
\end{tabular}

The search for optimal conditions, both in full-scale and in a model experiment, is one of the most common scientific and technical problems. They arise at the moment when the possibility of carrying out the process is established, and it is necessary to find the best (optimal in a sense) conditions for its implementation.

As noted, the consumption of electrical energy $\mathrm{W}$ of air coolers is a function

$$
W=f(T, t, r, p)
$$

From (1), it can be seen that several independent variables affect the consumption of electrical energy by air coolers. Accordingly, we will use the multiple regression method. According to the generally accepted technique, we will use vector notation. We denote by $\boldsymbol{Y}$ a vector of observations consisting of $\mathrm{n}$ elements, by $\boldsymbol{X}$ - a matrix of independent variables of size $m$ by $n$, where $m$ is the number of independent variables, and $n$ is the number of observations. 
In this notation, the problem can be formulated as follows

$$
Y=X b+e,
$$

where $e$ is independent random errors with mean 0 , which are interpreted as observational errors, and $b$ is a vector of unknown parameters that need to be estimated. The estimates of parameters $b$ will be denoted by $B$.

In our case, the dependent variable is the amount of consumed electrical energy $(W, \mathrm{~kW} \cdot \mathrm{h})$, and the independent ones are the gas temperature $\left(T,{ }^{0} \mathrm{C}\right)$, gas pressure $(p, \mathrm{~Pa})$, outside temperature $\left(t,{ }^{0} \mathrm{C}\right)$ and relative humidity $(r, \%)$ air (i.e., all variables listed in Table 1$)$.

Due to the presence of uncontrolled and even uncontrolled input variables $X_{i}-(T, t, r, p)$, the change in the value of $Y(W)$ is random. Therefore, equation (2) does not give us an exact connection between the input and output of the object and is only conditional on the mathematical expectation of a random variable $Y$, i.e., the regression equation.

The coefficients of the regression equation are determined by the following system of equations

$$
\sum_{g=1}^{N}\left(Y_{g}-\bar{Y}_{g}\right)^{2}=\sum_{g=1}^{N}\left(Y_{g}-\sum_{i=0}^{d} b_{i} x_{i g}\right)^{2}=\min
$$

where $Y_{g}$ is experimental values of the output parameter obtained at the $g$-th point of the factor space; $d$ is the number of terms in the regression equation.

Figure 2 shows a histogram of the distribution of consumed electrical energy $(W)$. Figure 3 shows a peak-to-peak diagram for consumed electrical energy $(W)$.

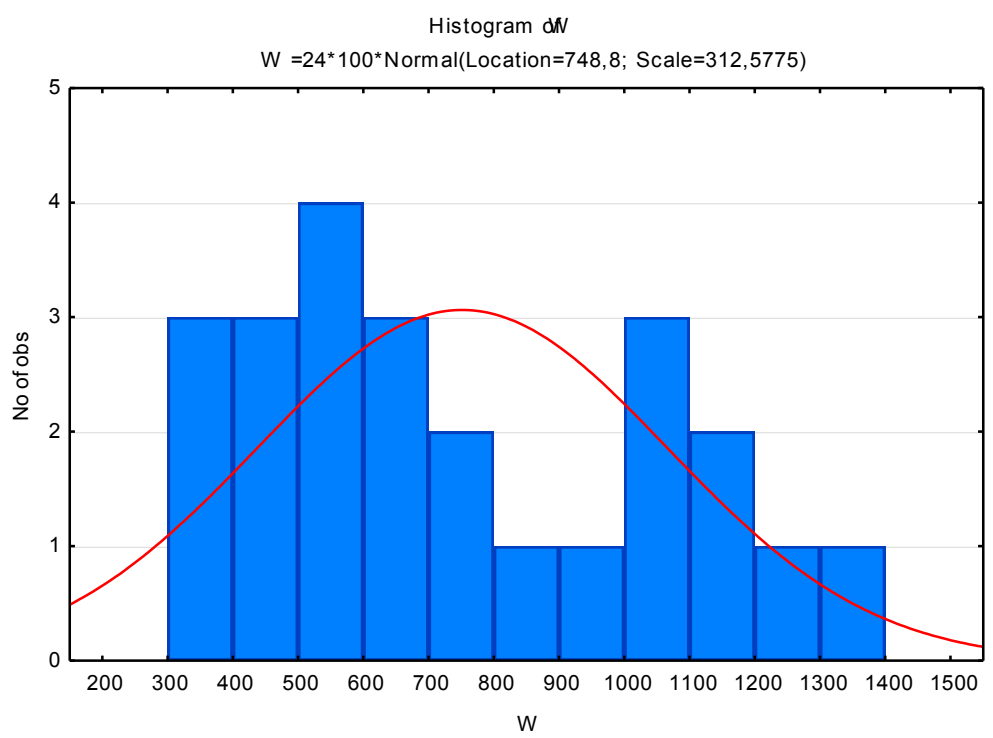

Fig. 2. Histogram of the distribution of consumed electrical energy 


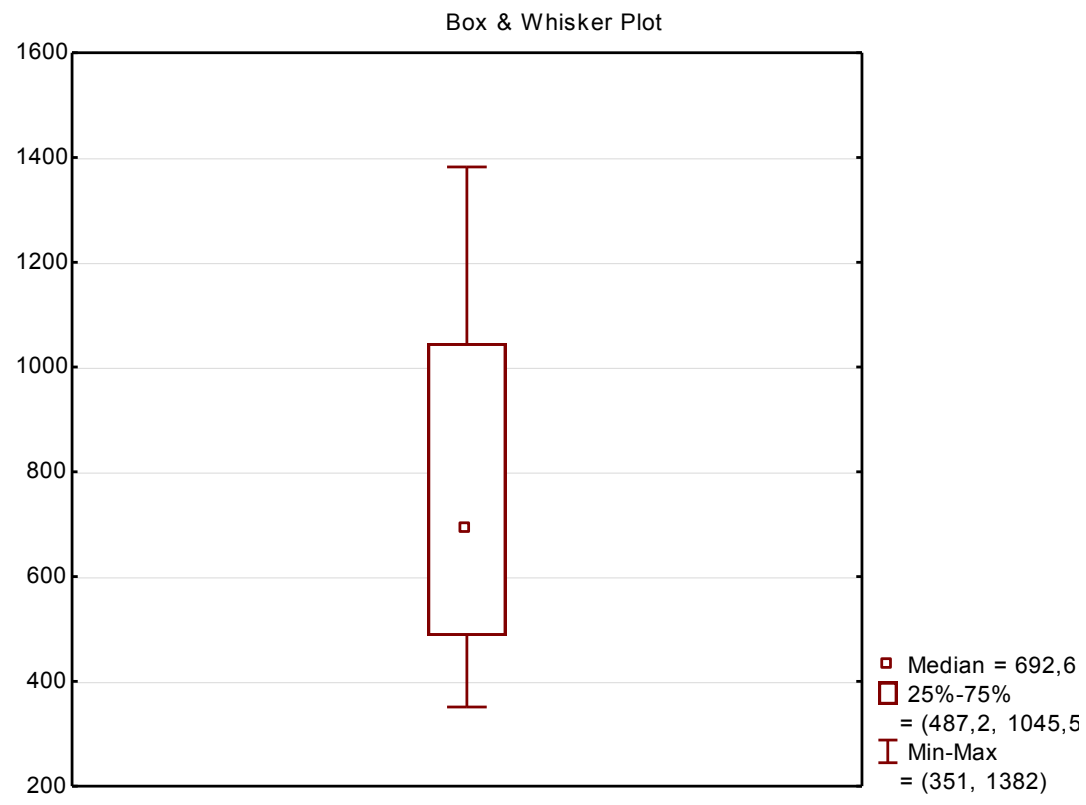

Fig. 3. Peak diagram for consumed electrical energy

To assess the degree of influence of the above factors on the power consumption of the gas air cooler, we use the Pareto diagram (Fig. 4.). In this diagram, the estimates of the ANOVA effects are arranged by the absolute value of the values: from the highest to the lowest. The magnitude of each effect is represented by a bar, and the bars are crossed with a line indicating how large the effect must be (that is, how long the bar must be) to be statistically significant. As shown in Figure 4, gas pressure exerts the greatest influence on the consumption of electrical energy (4.68). Based on the analysis of the experimental data, it was found that the degree of influence on the power consumption of the relative humidity of the air is insignificant (1.25).

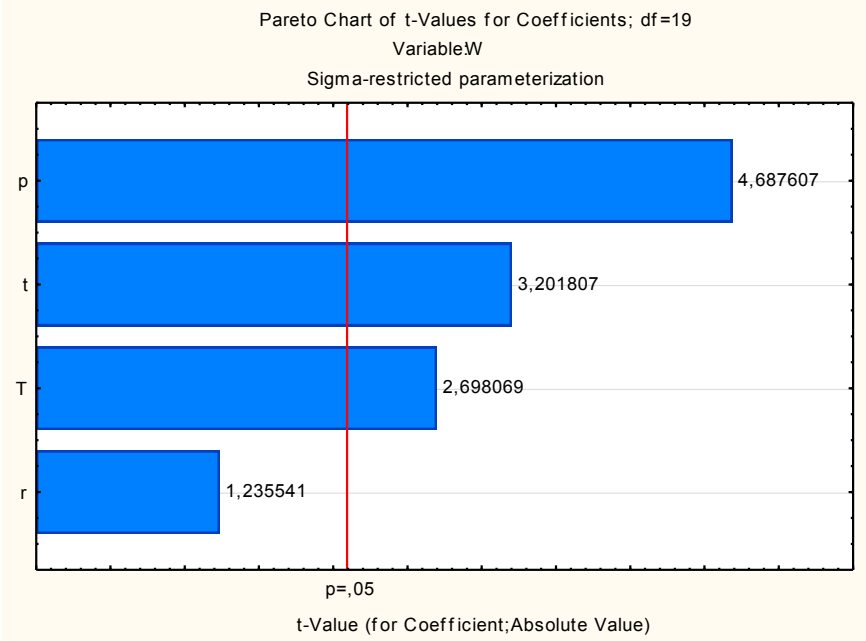

Figure 4. Pareto chart 
Table 2 shows the correlation matrix. The table shows that all factors are closely related.

Table 2. Correlation matrix

\begin{tabular}{|c|c|c|c|c|c|c|c|c|c|}
\hline \multirow[b]{2}{*}{ Effect } & \multicolumn{9}{|c|}{$\begin{array}{l}\text { Correlations of Vectors in Design Matrix X } \\
\text { Correlation matrix for the vectors } \\
\text { in the design matrix X }\end{array}$} \\
\hline & Level & Column & \begin{tabular}{l||} 
Effect \\
(F/R)
\end{tabular} & $\begin{array}{c}\text { Col. } 1 \\
\text { Intercpt }\end{array}$ & $\begin{array}{c}\text { Col. } 2 \\
t\end{array}$ & $\begin{array}{c}\text { Col. } 3 \\
\mathrm{r}\end{array}$ & $\begin{array}{c}\text { Col. } 4 \\
p\end{array}$ & $\begin{array}{c}\text { Col. } 5 \\
\mathrm{~T}\end{array}$ & $\begin{array}{c}\text { Col. } 6 \\
\text { W }\end{array}$ \\
\hline Intercept & & 1 & Fixed & & & & & & \\
\hline $\mathrm{t}$ & & 2 & Fixed & & $1,00000 C$ & $-0,81487 i$ & 0,75677 E & 0,953224 & $0,95803=$ \\
\hline$r$ & & 3 & Fixed & & $-0,81487 i$ & $1,00000 c$ & $-0,89318 i$ & $-0,87713 x$ & $-0,88187$ \\
\hline$p$ & & 4 & Fixed & & $0,75677 £$ & $-0,89318 i$ & $1,00000 c$ & 0,768641 & $0,87116 s$ \\
\hline $\mathrm{T}$ & & 5 & Fixed & & 0,953224 & $-0,87713 c$ & 0,768641 & 1,000000 & $0,95439 \mathrm{~s}$ \\
\hline $\mathrm{W}$ & & 6 & & & 0,95803 & $-0,88187\llcorner$ & 0,87116 ؟ & 0,95439 s & 1,000000 \\
\hline
\end{tabular}

The solution to the regression analysis problem should be divided into several stages: preliminary processing, selection of the type of regression equations, calculating the coefficients of the regression equation, checking the adequacy of the constructed function to the observation results.

Tables 3 and 4 allow you to get acquainted with the results of the analysis of variance of the regression equation. In the rows of the ANOVA table, the regression equations are the sources of variation: Regress. - due to regression, Residual - residual, Total - total. In the columns of the table: Sums of Squares is the sum of squares, df is the number of degrees of freedom, Mean Squares is the mean square, $F$ is the value of the F-criterion, p-level is the probability of the null hypothesis for the F-criterion.

Table 3. Parameter Estimates Sigma-restricted parametrization

\begin{tabular}{|c|c|c|c|c|c|c|c|c|c|}
\hline \multirow[b]{2}{*}{ Effect } & \multicolumn{9}{|c|}{$\begin{array}{l}\text { Parameter Estimates } \\
\text { Sigma-restricted parameterization }\end{array}$} \\
\hline & \begin{tabular}{|c|} 
W \\
Param. \\
\end{tabular} & $\begin{array}{c}\text { W } \\
\text { Std.Err }\end{array}$ & \begin{tabular}{c|}
$W$ \\
$t$
\end{tabular} & $\begin{array}{l}W \\
p\end{array}$ & $\begin{array}{l}-95,00 \% \\
\text { Cnf.Lmt }\end{array}$ & $\begin{array}{c}+95,00 \% \\
\text { Cnf.Lmt }\end{array}$ & $\begin{array}{c}\text { W } \\
\text { Beta (?) }\end{array}$ & $\begin{array}{c}\mathrm{W} \\
\text { St.Err.? }\end{array}$ & $\begin{array}{l}-95,00 \% \\
\text { Cnf.Lmt } \\
\end{array}$ \\
\hline Intercept & -24620, & $5091,10 ؛$ & $-4,8359<$ & 0,00011 t & -35276 & $-13964, \mathrm{f}$ & & & \\
\hline $\mathrm{t}$ & 19,5 & $6,09 \mathrm{c}$ & 3,20181 & $0,00469\{$ & 6,8 & 32,3 & 0,39519 s & $0,12342\{$ & 0,1368 \\
\hline$r$ & 11,9 & 9,604 & $1,2355<$ & 0,23168 s & $-8,2$ & 32,0 & 0,13579 & 0,10990 & $-0,0942$ \\
\hline$p$ & 589,3 & $125,71 E$ & 4,68761 & $0,00016 c$ & 326,2 & 852,4 & $0,38563 c$ & $0,08226 \epsilon$ & 0,2134 \\
\hline $\mathrm{T}$ & 57,1 & 21,167 & $2,6980 \bar{\imath}$ & $0,01424 \varepsilon$ & 12,8 & 101,4 & $0,40038 i$ & 0,14839 & 0,08978 \\
\hline
\end{tabular}

Table 4. Test of SS Whole Model vs. SS Residual

\begin{tabular}{l|c|c|c||c|c|c|c|}
\hline \multirow{3}{*}{$\begin{array}{l}\text { Dependent } \\
\text { Variable }\end{array}$} & \multicolumn{2}{|l|}{ Test of SS Whole Model vs. SS Residual } & & \\
\cline { 2 - 8 } & Multiple & Multiple & Adjusted & SS & df & MS & SS \\
R & R? & R? & Model & Model & Model & Residual \\
\hline W & $0,98840($ & $0,97693 !$ & 0,97207 & $219537 !$ & 4 & $548843, i$ & $51832,7 t$ \\
\hline
\end{tabular}

$\mathrm{F}$ - the criterion of the obtained regression equation is significant at the $5 \%$ level. The probability of a null hypothesis (p-level) is significantly less than 0.05 , which indicates the overall significance of the regression equation.

Thus, as a result of the performed regression analysis, the following equation of electrical energy consumption was obtained as a function of the outside air temperature $t$, relative air humidity $r$, gas pressure $p$, and gas temperature $T$

$$
W=19.5 t+11.9 r+589.3 p+57.1 T-24620.3 .
$$

\section{Conclusions}

As a result of the research, a model of electrical energy consumption by an air-cooled gas apparatus was determined, which was approximated by an equation with the original data set obtained during the experiment. Analysis of the model's compliance with the initial data allows us to speak about a sufficient degree of accuracy of the results obtained. The coefficients of the resulting linear regression equation are assessed as significant. The 
investigated model is assessed as adequate. The resulting model with a confidence interval of $95 \%$ describes the original data set.

\section{References}

1. Mochalin D and Titov V. Assessment and forecasting of energy consumption of a gas air cooling apparatus at a compressor station Pipeline transport: theory and practice $\mathbf{1}$ (47) 16-20, (2015)

2. Kalinin A F, Merkurieva Yu S and Fomin A V. Evaluation of the efficiency of using a frequency-controlled electric drive of gas air cooling devices at linear compressor stations of main gas pipelines Territory neftegaz 11 68-75, (2015)

3. Kolokolova E, Baikov I R, Burdygina E V and Smorodova O V. Influence of the angle of installation of the fan blades of air cooling devices on the temperature of the main gas outlet from the compressor station Innovations and investments 8 164-6, (2014)

4. Pashkin V V, Ivashkin O N and Shabanov V A. Method of controlling the frequencycontrolled drive of the gas air cooling apparatus with two-stage cooling Electronic scientific journal Oil and Gas Business 3 177-94, (2019)

5. Vasenin A.B. Analysis of technical and economic indicators of the use of gas air cooling apparatuses at compressor stations Automation and IT in the oil and gas area 3 (33) 10-9, (2018)

6. Mochalin D S and Titov V G. An invariant control system for the electric drive of the gas air cooling apparatus Electrical Engineering 8 27-32, (2015)

7. Abakumov A M, Alimov S V and Migacheva L A. Analytical and experimental study of stationary operating modes of gas cooling units of compressor stations of main gas pipelines Bulletin of the Samara State Technical University. Series: technical sciences 3(28) 113-7, (2010)

8. Ishnazarov $\mathrm{O}$ and Koroli M. Combined heat supply "heat pump-solar plant" system $J$. Phys.: Conf. Ser. 1691, (2020), 012051 doi:10.1088/1742-6596/1691/1/012051 